\title{
Maternal separation delayed the timing of GABA switch and induced the behavior disorders in male mice
}

\author{
Tomoaki Sato $^{1}$, Sayuri Yamanishi ${ }^{1}$, Minami Furukawa $^{1,2}$, Kazuo Tomita², Shoichi Miyawaki $^{2}$ \\ ${ }^{1}$ Applied Pharmacology, Graduate School of Medical and Dental Sciences, Kagoshima University, Japan, ${ }^{2}$ Orthodontics \\ and Dentofacial Orthopedics, Graduate School of Medical and Dental Sciences, Kagoshima University, Japan
}

Child neglect is an emerging social problem. Although it is reported that early life stress leads to psychiatric disorder, the cause is unclear. Early in life, the function of GABA (gamma-aminobutyric acid), an inhibitory neurotransmitter, changes from excitability to inhibitory (GABA switch). This GABA switch is mainly mediated by the up-regulation of KCC2 (K-Clcotransporter). Moreover, the precise timing of GABA switch is indispensable for constructing normal neural circuits. Therefore, the purpose of this study is to examine the influence of early life stress on higher brain functions. Neonatal C57BL/6J male mice were divided into control group and experimental group. Experimental group was subjected to maternal separation for 3 hours a day from postnatal day 1 (P1) to P21. Then both groups were weaned at P22. We analyzed KCC2 expression at hippocampus. Next, we analyzed the timing of GABA switch by Ca2+imaging method. Behavioral analyzes were conducted from P35. KCC2 was decreased at P7, P14 and P35and the timing of GABA switch was delayed in experimental group. We also found cognition and attention defects in experimental group by behavioral analyzes. Taken together, maternal separation inhibited the expression of KCC2 for a long lasting period and delayed the timing of GABA switch. 